\title{
A supplementary experiment for Matthews (2011, Can we use verbal estimation to dissect the internal clock? Differentiating the effects of pacemaker rate, switch latencies, and judgment processes. Behavioural Processes, 86, 68-74.)
}

\author{
William J. Skylark*
}

July 19, 2021

\begin{abstract}
Matthews (2011) examined the use of verbal estimation in studies of time perception. During the process of archiving the data for the three experiments reported in that paper, I realised that I had conducted an earlier version of Experiment 1; the method was very similar to the published version, but a larger set of to-be-judged durations was used. The pattern of results is the same as in the published version (although slightly noisier, with fewer trials per cell of the design). I report this earlier study here for the sake of completeness; I do not currently intend to submit this report to a journal.
\end{abstract}

Time perception researchers often posit the existence of some form of "internal clock", the operation of which underlies subjective time and is sensitive to a variety of nontemporal variables (see e.g., Matthews \& Meck, 2016, for an overview). Core components of this framework include a pacemaker that produces pulses which flow into an accumulator, and a switch that controls when the accumulation of pulses begins and ends (some theorists posit other components, too; e.g., Zakay \& Block, 1997). In this simple framework, a non-temporal variable that affects time judgments might do so by changing the latency of the switch. For example, if the latency to close the switch (to begin timing) is shortened and there is no change in latency open the switch (to end timing), more pulses will accumulate and subjective time will be lengthened. Alternatively (and not exclusively), the non-temporal factor might increase the rate of the pacemaker, so that more pulses flow into the accumulator per unit of physical time.

These two types of effect differ in their sensitivity to changes in the duration of the to-be-judged interval. A switch effect is expected to produce a uniform increase or decrease in the number of accumulated pulses; a pacemaker effect will become more pronounced as the time interval increases. Assuming that the mean number of pulses accumulated grows linearly with physical time, when subjective time is plotted against physical time a switch effect is expected to produce a change in intercept whereas a pacemaker effect will produce a change in slope.

One common technique for testing these possibilities involves having people make verbal estimates (in milliseconds) of a range of short durations (typically c. $80 \mathrm{~ms}-$ $1500 \mathrm{~ms}$ ). For example, Wearden et al. (1998) found a

*Department of Psychology, University of Cambridge. w.j.skylark@cantab.net. slope effect for sensory modality (the judgment function is approximately linear with a similar intercept for tones and lights, but a steeper slope for the former); similarly, Matthews et al. (2011) found that increasing the difference in intensity between a stimulus and its background leads to a slope effect (see also Wearden et al., 2007).

The conventional interpretation of these kinds of results in terms of switch and pacemaker effects requires the assumption that a change in slope necessarily indicates a change in the rate of growth of subjective time that is, a pacemaker effect. But it is possible that slope effects could arise even if the underlying change in subjective time is uniform - for example, if the conversion of subjective time (number of accumulated pulses) into a numeric estimate is non-linear. More broadly, at the time when the present experiment was conducted, no study (that the author was aware of) had produced a "pure" intercept - that is, all attempts to use verbal estimation to dissect the internal clock had found evidence for pacemaker changes. These considerations raise the prospect that the verbal estimation task and analysis is unable to detect a switch effect.

Against this background, Matthews (2011) reported 3 experiments in which participants estimated the duration of blank intervals delimited by two briefly-presented markers. Each marker was the outline of a black square on a white background. In Experiment 1, the key manipulation was of the size of the second square: on some trials the second marker was only slightly different in size from the first marker (a "small jump"); on other trials, the second marker was very different in size (a "big jump"). The logic of this design was that, if the only thing that changes between two trials is the size of the end marker, there should be no possibility of a slope effect because the time-varying blank interval that has to be judged is iden- 
tical in both conditions. Correspondingly, if a slope effect were found, it would imply that the conventional identification of slope effects with pacemaker changes might need reconsideration.

During the process of archiving the data from the 3 experiments reported by Matthews (2011), I realised that an earlier version of Experiment 1 was also conducted. The logic and design is the same as in the published paper, as is the pattern of results. The only notable difference was the use of a larger set of to-be-judged durations. That experiment is reported here.

\section{Method}

The Materials and Data are available from: https://osf.io/kx8hq/

\section{Participants}

Twenty six participants took part for a payment of $£ 4$. Two were excluded without further analysis because they produced a large proportion of ineligible responses $(25 \%$ and $100 \%$, of a mean of $1.2 \%$ across the other participants). Demographic data are not available.

\section{Stimuli}

The stimuli were the same 4 black empty squares as used in Experiment 1 of Matthews (2011); see that paper for further details. The squares had sides measuring 40, 60, 150 , and 170 pixels; they were labelled $1-4$, respectively.

\section{Design and Procedure}

The design and procedure were virtually identical to Experiment 1 of Matthews (2011), as was the testing equipment. Participants took place in sound attenuating cubicles; the stimuli were presented on CRT monitors with a refresh rate of $85 \mathrm{~Hz}$. (See Matthews, 2011, for further details.)

On each trial participants saw two squares, one after the other, and had to judge the duration of the blank interval between them. They typed their estimate of the duration, in milliseconds, and were reminded at the start that $1000 \mathrm{~ms}=1$ second (so that, for example, "if the blank interval between the squares lasts one tenth of a second, type 100 , if it lasts 1.25 seconds, type 1250 , and so on"). They were also told that none of the durations would be less than 50 ms or more than 2000 ms.

The experiment had a 10 (duration) $\times 2$ (jump size: small, big) $\times 2$ (size of first marker: small, large) mixed design, with duration and jump size as within-subject factors and marker order as a between-subjects factor. For participants in the small marker first condition the first square was always square 1 (the smallest); the second marker was then square 2 on the small-jump trials and square 4 on the large-jump trials. For participants in the large marker first condition, the first square was always square 4 (the largest); the second marker was then square
3 (on the small-jump trials) and square 1 (on the largejump trials). Participants completed 5 blocks of 20 trials, with one occurrence of each duration-jump size combination per block (in random order).

Presentation was controlled by DMDX (Forster and Forster, 2003). The sequence of events on each trial was: blank interval for 1 second, first marker for $259 \mathrm{~ms}$ (22 frames), target interval (blank screen) for 7, 17, 28, $38,49,59,70,80,91$, or 101 frames (corresponding to $82,200,329,447,576,694,824,941,1071,1188 \mathrm{~ms}$ ), second marker for 22 frames (259 ms), response window (participants could take as long as they liked over their response). Participants could not see what they typed (no values appeared on-screen), but they were told if they made a mistake they should press the $Q$ key and then re-type their response.

\section{Results}

A handful of trials were excluded because the participant made an ineligible response (less than 50 ms or more than $2000 \mathrm{~ms}$ ), or because the software indicated a (possible) display timing error. Across the final sample of 24 participants, the overall proportion of excluded trials was $2 \%$.

Figure 1 plots the mean responses for each cell of the design. The top panel shows the results for participants for whom the first marker was small; the middle panel shows shows for participants for whom the first marker was large; the bottom panel shows the results collapsed across the between-subjects factor. As might be expected given the small number of trials at each duration, the pattern is somewhat noisy.

Submitting the mean responses to a $2 \times 10 \times 2$ ANOVA indicated a sizeable effect of stimulus duration: as one would expect, people gave larger estimates for longer durations $F(1.80,39.59)=64.05, p<.001$, $\eta_{p}^{2}=.74$ (here and elsewhere, following the approach taken in Matthews, 2011, a Huynh-Feldt correction has been applied when sphericity is violated). There was also a notable effect of jump size: the durations were judged to be longer when the jump in size was bigger, $F(1,22)=24.11, p<.001, \eta_{p}^{2}=.52$, and this effect was independent of duration, $F(4.14,90.99)=1.62$, $p=.174, \eta_{p}^{2}=.07$. None of the other effects were "significant": the effect of first marker size, $F(1,22)=0.65$, $p=.430, \eta_{p}^{2}=.03$; interaction between first marker size and jump size, $F(1,22)=0.93, p=.345, \eta_{p}^{2}=$ .04; interaction between first marker size and duration, $F(1.80,39.59)=0.19, p=.807, \eta_{p}^{2}=.01$; three-way interaction, $F(4.14,90.99)=0.95, p=.441, \eta_{p}^{2}=.04$.

Following the example of previous work, for each participant the mean judgment was regressed on stimulus duration; this was done separately for the small jump and large jump conditions. The mean intercepts (with SDs in parentheses) for participants in the small first marker condition were $12.84 \mathrm{~ms}$ (223.42) for the small-jump condition and $88.99 \mathrm{~ms}$ (188.02) for the big-jump condition; for those in the large first marker condition the corresponding values were $69.34 \mathrm{~ms}$ (311.46) and 161.72 (268.21). The mean slope parameters for participants in the small first 

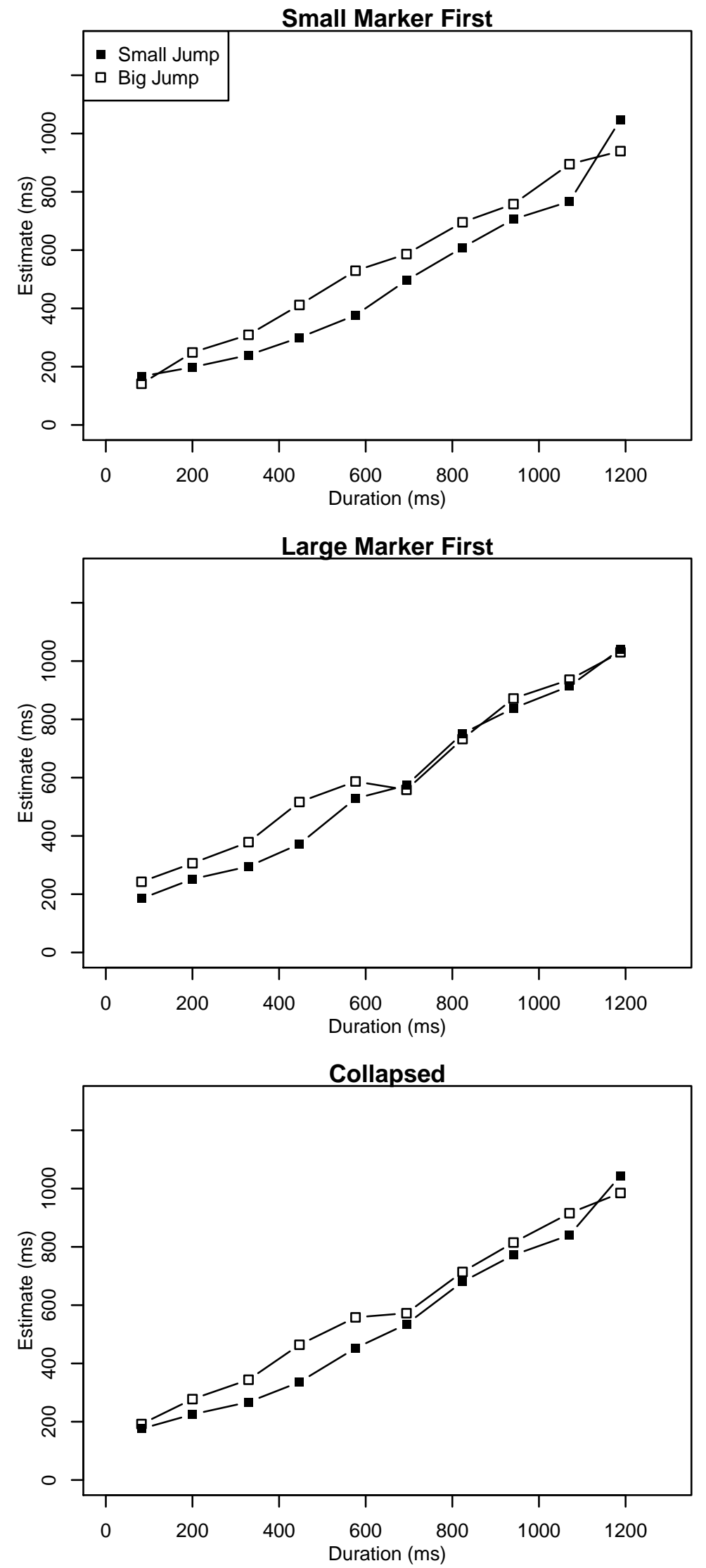

Figure 1: Mean responses from the small marker first condition (top panel), the large marker first condition (middle panel) and collapsed across conditions (bottom panel). 
marker condition were 0.753 (0.441) for the small-jump condition and 0.726 (0.292) for the large-jump condition; for those in the large marker first condition the corresponding means were $0.796(0.494)$ and $0.715(0.370)$, respectively.

The resulting intercepts and slope coefficients were submitted to $2 \times 2$ ANOVAs with jump size as a withinsubjects factor and first marker size as a between-subjects factor. (Arguably, a mixed effects model would be better but I have used the approach taken in Matthews, 2011, and earlier work for the sake of comparability.) The analysis of intercepts indicated a substantial effect of jump size, $F(1,22)=9.95, p=.005, \eta_{p}^{2}=.31$, with no effect of first marker size and no interaction, $F(1,22)=0.42, p=$ $.522, \eta_{p}^{2}=.02$ and $F(1,22)=0.09, p=.764, \eta_{p}^{2}=.00$, respectively. In contrast, the analysis of the slope coefficients indicated little effect of jump size $F(1,22)=1.37$, $p=.254, \eta_{p}^{2}=.06$, again with no meaningful effect of first marker and no interaction, $F(1,22)=0.01$, $p=.921, \eta_{p}^{2}=.00$ and $F(1,22)=0.35, p=.562$, $\eta_{p}^{2}=.02$, respectively.

\section{Discussion}

This pattern of results is the same as that reported in Experiment 1 of Matthews (2011) (although the present data are somewhat noisier): the intervals were judged longer when the jump in marker size was larger, but there was little indication that this effect was modulated by the duration of the to-be-judged interval. That is, varying the size of the second marker produced an intercept effect but not a slope effect. The potential implications of this pattern was discussed by Matthews (2011), who also reports two further studies of the effects of marker size on the intercept and slope of the judgment function. Readers are referred to that paper for further information.

\section{References}

Forster, K.I., \& Forster, J.C. (2003). DMDX: A windows display program with millisecond accuracy. Behavior Research Methods, Instruments, \& Computers, 35, 116--124.

Matthews, W.J. (2011). Can we use verbal estimation to dissect the internal clock? Differentiating the effects of pacemaker rate, switch latencies, and judgment processes. Behavioural Processes, 86, 68-74. https: //doi.org/10.1016/j.beproc.2010.09.006

Matthews, W.J., \& Meck, W.H. (2016). Temporal cognition: Connecting subjective time to perception, attention, and memory. Psychological Bulletin, 142(8), 865-907. https://doi.org/10.1037/bul0000045

Wearden, J.H., Edwards, H., Fakhri, M., \& Percival, A., (1998). Why "sounds are judged longer than lights": Application of a model of the internal clock in humans. Quarterly Journal of Experimental Psychology, 51B(2), 97-120. https://doi.org/10.1080/713932672

Wearden, J.H., Norton, R., Martin, S., \& Montford-Bebb, O. (2007). Internal clock processes and the filled- duration illusion. Journal of Experimental Psychology: Human Perception and Performance, 33, 716-729. https://doi.org/10.1037/0096-1523.33.3.716

Zakay, D., \& Block, R.A. (1997). Temporal cognition. Current Directions in Psychological Science, 6, 12-16. https://doi.org/10.1111/1467-8721. ep11512604 